\title{
EVALUACIÓN DEL USO DE TECNOLOGÍAS EN LA ENSEÑANZA UNIVERSITARIA A DISTANCIA DE LA UNED
}

\section{(EVALUATION OF THE USAGE OF TECHNOLOGIES AT UNED DISTANCE EDUCATION UNIVERSITY)}

\author{
Emilia Carmena Yáñez
}

Javier Navarro Fernández

Universidad Nacional de Educación a Distancia, UNED (España)

\section{RESUMEN}

El presente trabajo analiza las nuevas tecnologías que ofrece la Universidad Nacional de Educación a Distancia (UNED), y el uso que de ellas realizan los estudiantes de esta universidad, con el objetivo de analizar los datos obtenidos, de manera que nos permitan sugerir conclusiones y propuestas de mejora que aumenten el clima de confianza y apoyo entre los distintos estamentos de la universidad; además, de servir de base para sucesivos trabajos de investigación.

Nuestro estudio será descriptivo-cuantitativo, en un primer momento, y comparativo posteriormente, ofreciendo las opiniones recogidas de un amplio número de estudiantes; utilizaremos como instrumento de recogida de datos un cuestionario aplicado a dicho colectivo.

El tema central del artículo aborda la problemática de las nuevas tecnologías ofrecidas por la UNED. La tabla ANOVA, junto con la prueba Chi-cuadrado de Pearson, $\chi^{2}$, nos indicará si la distribución F presenta diferencias significativas al 95\% de confianza.

Palabras clave: tecnologías de la información, campus virtuales, redes digitales, plataforma aLF, WebCT, e-learning.

\begin{abstract}
This paper analyses the new technologies provided by Spain's Distance Learning University (UNED) and their usage by UNED students. The objective of this paper is to evaluate the collated date so as to be able to draw conclusions and make recommendations for improvement proposals that will lead to increased trust and support among the various sections of the university, while providing a basis for subsequent research work.
\end{abstract}


Our study is primarily descriptive and quantitative. Secondly, it is comparative as it provides opinions that are gathered from a large group of students. For data gathering instruments, we used data from a survey that was applied to the aforesaid group.

The core theme of the article examines the difficulties that are experienced using the new technologies at UNED. The ANOVA table, along with Pearson's Chi-squared, $\chi^{2}$, Test, will indicate if the $\mathrm{F}$ distribution shows significant differences in comparison with the $95 \%$ degree of confidence.

Keywords: information technology, virtual campuses, digital networks, Alf platform, WebCT, E-learning.

En líneas generales la enseñanza a distancia, entendiendo por ello la educación no presencial sin contacto físico entre docente y alumno, existe desde hace más de medio siglo en numerosos países tanto europeos como en los Estados Unidos de América $^{1}$, siendo en este país, siglo XIX, cuando comienza su andadura ante la creciente demanda de emigrantes norteuropeos, por la necesidad de querer que sus hijos recibieran la formación necesaria de docentes que residían en sus países de origen, con el fin de que fuesen preservadas sus costumbres y culturas originales, empleando para ello los sistemas postales tradicionales.

Posteriormente, a mediados del siglo XX, y ante la llegada de nuevos medios de comunicación, como la radio y la televisión, es cuando la enseñanza universitaria a distancia recibe un gran impulso al aplicar sonido y visión a este tipo de enseñanza, siendo reclamada por miles de ciudadanos de todo el mundo con el objetivo de poder alcanzar una formación que les permita conseguir una profesionalización que de no ser por este tipo de enseñanza les resultaría prácticamente imposible a muchos de ellos.

Debido a esa demanda social y a la evolución tecnológica de las Tecnologías de la Información y de la Comunicación (TIC), cuyo uso fue aprobado por la UE en marzo de 2000, basadas fundamentalmente en los ordenadores (hardware, software, redes, bases de datos,...), se brindó la posibilidad de ofrecer una enseñanza universitaria a distancia, abierta y rápida, mediante un sistema de comunicación multimedia que permitiera poder mejorar el aprendizaje de numerosos estudiantes, cobrando así un fuerte impulso este tipo de enseñanza que en un principio parecía inimaginable.

También la UNESCO ${ }^{2}$ hizo hincapié en la importancia del uso de las nuevas tecnologías en la enseñanza universitaria a distancia recomendando el fortalecimiento de la cooperación internacional en dicho campo. Posteriormente se analizaron las experiencias internacionales sobre la utilización de las TIC en los sistemas educativos, 
examinando los resultados satisfactorios obtenidos por las mismas, en la enseñanza universitaria a distancia.

Diversos autores apuntan en esta dirección así, y por citar alguno de ellos, Sevillano García (1998b) indica como una de las principales preocupaciones de todos los sistemas educativos la incorporación a la práctica docente de las nuevas tecnologías, mientras que Area (2002) considera de gran importancia las Tecnologías de la Información y Comunicación (TIC), ya que cada día, dentro de la sociedad actual, existe una gran parte de la población que está en contacto con ella.

En la actualidad la enseñanza universitaria a distancia es una exigencia social que está alcanzado un papel relevante en la oferta formativa de los países desarrollados occidentales, intentando evitar desigualdades sociales y económicas entre ciudadanos, trabajadores, profesionales o estudiantes, que por diversos motivos de tiempo o lejanía, no han podido cursar estudios en las formas tradicionales de asistencia a un centro educativo.

Basta con echar una mirada a los principales países de la Unión Europea con tradición en la enseñanza universitaria a distancia, tanto pública como privada, para hacernos una idea general del largo camino recorrido por este tipo de enseñanza. Las nuevas tecnologías de la información y comunicación (TIC), conducen a la creación de los campus virtuales, (Van Dusen 1997) ${ }^{3}$, los cuáles, a través de las redes digitales, nos muestran una enseñanza universitaria a distancia cada vez más potente y con la capacidad de llegar a cualquier lugar del mundo.

De esta manera se observa como las nuevas generaciones cada vez utilizan más en sus estudios el empleo de las TIC con el objetivo de universalizar sus conocimientos, obteniendo mayor calidad en su aprendizaje, además de propiciar el desarrollo de hábitos, habilidades y destrezas para la búsqueda selección y tratamiento de la información.

\section{UNIVERSIDAD NACIONAL DE EDUCACIÓN A DISTANCIA, UNED}

Durante la reforma educativa de los años 70 y con el objetivo de conceder la igualdad de oportunidades para todos, el Gobierno español se vio en la necesidad de crear una institución en nuestro país que, sin perder su carácter académico y científico, ofreciera una enseñanza universitaria a distancia de calidad que diese una respuesta adecuada a la demanda social existente en el momento. 
Esa demanda existente sobre enseñanza universitaria a distancia propició la creación en nuestro país de la Universidad Nacional de Educación a Distancia (UNED), año 1972, conforme la enseñanza que impartía otra Universidad Europea como era la Open University. Así lo confirma la Ley de Reforma Universitaria, (LRU 1983).

Como todo tipo de enseñanza universitaria a distancia, la característica más evidente de la UNED es la ausencia de una relación presencial cotidiana entre profesores y estudiantes, estando su modelo educativo basado en las nuevas teorías sobre el aprendizaje, considerando que la relación directa entre profesor y alumno no es imprescindible, ya que es posible aprender en solitario determinados contenidos, siempre que exista una metodología adecuada que garantice la calidad y claridad de los mismos.

Esta característica unida a unos medios pedagógicos y tecnológicos adecuados, como son una comunicación fluida entre profesor y alumno, para consultas y asesoramiento, dan garantías suficientes para lograr una enseñanza universitaria a distancia de calidad.

De este modo, y a lo largo de sus 40 años de impartición de enseñanza universitaria a distancia, esta Universidad se ha ido adaptando a las necesidades tecnológicas que le han permitido ser pionera en este tipo de enseñanzas, no sólo en nuestro país sino también fuera de nuestras fronteras.

En un primer momento, se creó la Unidad de Virtualización Académica (UVA) para, a través de ella, conseguir el diseño virtual de los contenidos necesarios que permitiesen adquirir conocimientos a través de la "Red", pudiendo los estudiantes acceder a ese entorno tecnológico desde cualquier lugar del mundo y en cualquier momento, sin coincidencia en el tiempo ni en el espacio con compañeros y profesores.

De esta forma los estudiantes pueden gestionar mejor su tiempo, ahorrándose desplazamientos y al mismo tiempo acostumbrarse a unas herramientas que le serán de una vital importancia en el futuro, tanto en sus estudios como en su trabajo.

Posteriormente en esta universidad, y con el desarrollo de las telecomunicaciones, se han creado los “cursos virtuales", los cuáles también facilitan el seguimiento de la enseñanza universitaria a través de Internet, "on line", en aquellas materias donde el número de estudiantes no alcanza el mínimo necesario establecido por dicha universidad para impartir tutorías presenciales de las mismas. 


\section{LA EVALUACIÓN Y LAS TIC EDUCATIVAS}

Considerando a las Tecnologías de la Información y Comunicación el instrumento idóneo para apoyar y facilitar el intercambio de información y comunicación entre personas y organizaciones, creemos que las mismas optimizan y mejoran los procesos educativos, desarrollando tareas y contribuyendo a la competitividad de las universidades.

Así, el uso, o no, de estas tecnologías de información trae como consecuencia el incremento de las diferencias entre los países desarrollados y los emergentes, por lo que pensamos que su uso continuado impulsará los procesos educativos.

Dentro de estos procesos, quizás sea la evaluación el proceso educativo más unido a la educación desde sus comienzos, llevando a cabo una transformación positiva debida, fundamentalmente, a la renovación de los medios y sus más que apropiados procesos que permiten evaluar la enseñanza a distancia.

Todo ello no habría sido posible sin las TIC, ya que su uso ha facilitado este tipo de enseñanzas con resultados francamente positivos, entre otros, su aprovechamiento para la evaluación de estas enseñanzas, contribuyendo a la mejora de la calidad educativa así como a la toma de decisiones más coherentes y fundamentadas, dando una gran solidez a todo el proceso educativo a distancia.

\section{Como indicó Williams (2000):}

como cualquier educador sabe en el 2001, la tecnología está jugando una parte importante en los esfuerzos por reformar la educación. Pero la tecnología en sí misma no será suficiente para lograr los deseados cambios. También debe producirse un cambio en la pedagogía, que incluya el uso de las mejores prácticas de enseñanza y el constructivismo.

Actualmente y en un mundo tan globalizado como el actual, la práctica de la evaluación se hace cada vez más necesaria y común, tratando de que ésta no sea solamente una práctica realizada cada cierto tiempo, generalmente en las aulas, y con ánimos calificadores, sino que ha ido expandiéndose apareciendo otros modelos de enseñanza que, ya desarrollados, han dado lugar a un nuevo modelo de gestión.

Y es en este nuevo modelo que la evaluación tiene una función primordial como elemento de información valorativa sobre el estado de la educación, contribuye a tomar las mejores decisiones posibles, en cuanto coherentes y profundas, mejorando la calidad de la enseñanza a distancia, mediante un sólido sistema de evaluación de la calidad de la educación, entendiendo por ella la que logra hacer que los estudiantes 
aprendan lo que realmente tienen que aprender es decir, lo que figura en el programa de sus asignaturas.

Todo ello no sería posible sin que hubiese unos equipos docentes lo suficientemente preparados para poder ofrecer a sus estudiantes una enseñanza de calidad, así como la existencia de la elaboración, por parte de los mismos, de unos materiales y guías didácticas, claras y concisas con lo que los docentes pretenden de sus estudiantes, así como acordes y adecuadas para el uso de la enseñanza a distancia.

Dado que a la hora de aprender los estudiantes deben superar una serie de obstáculos, el docente debe antes identificarlos para, una vez evaluadas las causas de dichos obstáculos, y después de analizarlos, ayude a los estudiantes a superar los mismos.

De ahí, que una de las funciones de la evaluación sea regular los aciertos y errores habidos, de manera que en un futuro los estudiantes progresen en sus conocimientos haciendo lo más efectivo posible sus estudios, siendo necesario para ello la adecuación correcta del proceso didáctico a los progresos de aprendizaje de los estudiantes, ofreciéndoles la ayuda pedagógica necesaria para ajustarse a sus necesidades educativas.

Las universidades de educación a distancia, a partir de la implantación de Internet, han introducido las Nuevas Tecnologías de la Información y Comunicación (NTIC) en sus enseñanzas, lo que ha originado unos importantes cambios en su estructura y organización educativa, haciendo que ésta derive hacia modelos de configuración en Red como la alternativa más adecuada a los formatos más tradicionales.

En este sentido podemos indicar que "la historia demuestra que la introducción de las nuevas tecnologías generalmente va acompañada de unos cambios importantes en la organización del trabajo" (Battes, 2001, p.19).

\section{TECNOLOGÍAS, MEDIOS Y RECURSOS EN LA UNED}

Sus objetivos principales consisten en el desarrollo de nuevas aplicaciones de enseñanza y aprendizaje, incluyendo nuevas versiones de las plataformas y servicios básicos utilizados. Además, está centrada en temas de investigación que permitan afrontar el futuro del uso adecuado de las TIC para potenciar los procesos de enseñanza y aprendizaje en una universidad de educación a distancia como la UNED. 
En este sentido los cursos virtuales de la UNED pretenden ampliar los servicios prestados por la institución a los estudiantes, así como aprovechar las posibilidades de Internet para profundizar en la metodología de la enseñanza a distancia.

$\mathrm{El}$ acceso se realiza mediante clave personal y password, a través de enlace en el portal: UNED http://www.uned.es, o desde el servidor específico http://virtual. uned.es/, siendo la plataforma elegida la WebCT ${ }^{5}$, (http://www.webct.com), que se encuentra en el servidor de los cursos virtuales de la UNED. Para trabajar en ella es necesario estar previamente autenticado.

\section{WEBCT}

Una vez que accedemos a la WebCT, ésta nos proporciona los siguientes servicios:

- Información sobre los cursos virtuales en WebCT.

- Acceso a los cursos virtuales de la UNED para usuarios de enseñanzas regladas y no regladas.

- Acceso a los cursos virtuales de la UNED como alumnos de enseñanzas no regladas y alumnos y administradores de instituciones externas.

Cuando se entra en la asignatura que hemos seleccionado, hay distintas herramientas para trabajar en la misma. El procedimiento es el que figura a continuación.

- "Portal de la asignatura". Espacio desde el que se puede acceder a las aplicaciones para el seguimiento del curso. En este espacio se incluyen distintas herramientas que el profesor elige de entre las opciones que soporta la plataforma, siendo las más habituales:

- "Herramientas de contenidos". Aplicaciones que sirven para complementar los contenidos de los materiales escritos.

- "Herramientas de comunicación". Mediante esta herramienta podemos recibir directamente los comunicados o circulares que el equipo docente de la sede central distribuye durante el curso. También disponen de foros y chat para la interacción con compañeros, profesores y profesores-tutores. 
- "Herramientas de estudio". Este tipo de herramientas brinda estrategias metodológicas específicas de e-learning y permite aportaciones de estudiantes en espacios ad hoc, además de la formación de grupos de trabajo.

- "Herramientas de evaluación y auto-evaluación". Permiten el poder realizar seguimientos del proceso de aprendizaje del estudiante, por parte de profesores y del propio estudiante. El principio pedagógico es el propio de la evaluación procesual, formativa, si bien es el docente quien elige el método concreto de aplicar las herramientas.

\section{aLF}

La plataforma de e-learning aLF se viene desarrollando desde el año 2000, siendo Innova la encargada, en su labor de I+D dentro de la UNED, de ofrecer un producto ${ }^{6}$ GPL sólido, potente y ampliamente utilizado en todo el mundo.

- aLF es una plataforma que permite impartir y recibir formación de los distintos grados del EEES, así como gestionar y compartir documentos, crear y participar en comunidades temáticas, realización de proyectos online... Aunque está desarrollada específicamente para la comunidad de profesores y alumnos de la UNED, es también accesible al público en general.

La plataforma, esencialmente, dispone de tres niveles que resumimos a continuación:

- Mi portal, área general de trabajo de la plataforma en sí, desde donde puede dirigirse al resto de niveles. Estará personalizada para cada usuario con sus datos, con los cursos en los que está matriculado y zonas a las que puede acceder.

- Curso o Comunidad

- Cursos: figura la docencia impartida; licenciatura, grados, formación continua... o en su lugar los estudios en los que se esté matriculado.

- Comunidad: aparecen las comunidades a las que pertenece como docente: Profesor UNED, Tutor, TAR (Tutor Apoyo Red), Redes de investigación para la labor docente...

- Grupo de trabajo, Se pueden crear dentro de un Curso, o englobado en una Comunidad 
Dicha plataforma nos facilitará hacer un buen uso de los recursos disponibles en Internet para paliar las dificultades que ofrece el modelo de enseñanza a distancia. Con este objetivo la UNED pone a disposición, tanto de los equipos docentes como de los estudiantes, las herramientas necesarias para que encuentren la manera de compaginar el trabajo individual con el aprendizaje cooperativo.

Para abordar estos retos la UNED no sólo se basa en una tradición de 40 años en el uso de los distintos medios disponibles que facilitan los procesos de enseñanza y aprendizaje, sino que más recientemente ha establecido el Centro de Innovación y Desarrollo Tecnológico (cInDeTEC), como una de las nuevas herramientas desarrolladas por la UNED, que permiten ampliar los servicios ofrecidos para potenciar los propios procesos de enseñanza y aprendizaje, permitiendo, por un lado y de forma general, abordar nuevas soluciones a los retos planteados por la llamada sociedad del conocimiento y, por otra parte y de forma más específica, dar respuesta a los nuevos objetivos de la Universidad en el denominado Espacio Europeo de Educación Superior, mucho más centrado en las necesidades individuales de los estudiantes.

Todo ello se ha ido fomentando desde la Sección de Innovación de la UNED, anteriormente conocido como Tec-Infor, (Unidad Técnica de Investigación y Formación en Recursos Tecnológicos), siendo la comunidad de docentes TAR, (Tutores de Apoyo en Red) una de las herramientas más importantes de la plataforma $a L F$. Dicha comunidad está orientada a los profesores de la sede académica de la UNED que por diferentes razones realizan las tareas asignadas, para el curso docente en vigor, a la figura del Tutor de Apoyo en Red. Los profesores participantes en esta Comunidad pueden:

- Consultar y descargar los "Documentos" básicos sobre las tareas del TAR.

- Consultar la "Agenda” para la planificación de las actividades del TAR.

- Consultar dudas y realizar comentarios sobre la figura y las tareas del TAR en los foros de debate que se encuentran en la página de "Inicio" de la Comunidad.

- Seguir las "Novedades" aparecidas en esta Comunidad, con las citas, tareas, documentos y mensajes remitidos en los últimos días.

- Realizar el "Envío de informes" mensuales y el informe final sobre la actividad desarrollada. 
De todos los medios audiovisuales que ofrece la UNED, son las Tecnologías Analógicas las más utilizadas. Entre ellas podemos destacar:

- Aulas AVIP7, proporcionan la "presencialidad virtual" que consiste en que desde cualquier centro o aula se puede acceder a las actividades presenciales de cualquier otro centro o aula.

Existen dos tipos de aulas AVIP:

- Aulas AVIP de Videoconferencia (Nivel 1): dotadas con sistemas de videoconferencia y pizarra digital interactiva, pizarra online, que mediante la Unidad de Control Multipunto (MCU) permite interconectar varios centros y aulas a la vez.

- Aulas AVIP de Webconferencia (Nivel 1+), aulas más virtuales, con webcam, equipo supresor de eco y pizarra con equipamiento pensado para trabajar directamente con herramientas en Internet, utilizando el software de conferencia online que permite realizar webconferencia de uno (docente) a muchos (alumnos) con roles diferenciados (moderador, presentador e invitado) a la hora de poder usar las funcionalidades disponibles (vídeo, audio, chat, pizarra, presentaciones, mostrar escritorio...).

- Cadena Campus, es el nivel 2 de servicio y consiste en la captura de las sesiones (seminarios, tutorías...) emitidas desde las aulas AVIP para su almacenamiento y difusión en directo y diferido por Internet.

- Tutorías en Línea, también denominado nivel 2+, y consiste en una aplicación integrada en la plataforma aLF que permite a los docentes y alumnos desarrollar una tutoría en línea desde cualquier lugar con conexión a Internet.

- Radio, es el medio audiovisual con mayor audiencia dentro de la UNED, por poder llegar a cualquier lugar de la geografía nacional, en donde el mensaje elaborado por el profesorado de la sede central se transmite de forma unidireccional con contenidos de distintas disciplinas, tanto en las enseñanzas regladas como en las de matrícula abierta.

Los contenidos de la programación, seleccionados y elaborados por profesores de la UNED, y otros especialistas colaboradores, se presentan en bloques bien definidos que se pueden escuchar a través de Radio 3 FM, Radio Nacional de España; 
las frecuencias de emisión, varían según cada comunidad autónoma. Se pueden consultar en la dirección: radio@uned.es.

- Televisión educativa, ofrece conjuntamente imagen y sonido, lo que le hace ser de gran interés, sobre todo, a alumnos de experimentación, tanto de campo como de laboratorios, donde sólo el medio sonoro se queda insuficiente.

La UNED cuenta con una televisión educativa abierta, que ofrece una programación semanal a través de la segunda cadena de TVE, conjugando temas que son propios de las enseñanzas de la UNED con aquellos que, de manera amplia, interesan a una comunidad más extensa.

Actualmente existe la plataforma TeleUNED, a la que se puede acceder desde el portal de la UNED, http://www.uned.es, o desde su propio servidor en, http://www. teleuned.com.

\section{OBJETIVO DE LA INVESTIGACIÓN}

El origen, los desencadenantes y las causas de los problemas de la enseñanza a distancia en la UNED, pueden tener una procedencia diversa, pero en su mayor parte están unidos a aspectos del trabajo cotidiano que demanda nuestra dedicación, de las vivencias personales y profesionales, de la experiencia, de reuniones de trabajo...

En el caso que nos ocupa, el objetivo se centra sobre el análisis y la valoración que los estudiantes de la UNED hacen de la misma. Surge de la confluencia de una serie de factores y situaciones personales que despertaron el interés inicial por el trabajo de investigación, y que, con posterioridad, a medida que se ha ido avanzando en su estudio inicial, nos ha permitido ir teniendo un conocimiento más profundo sobre el mismo.

Tomando como base la actual situación de cambio y transformación que se está dando en la enseñanza a distancia en general y en la UNED en particular, nos obligana preguntarnos si: ċla enseñanza a distancia ofertada por la UNED responde a las necesidades del colectivo de estudiantes, en cuanto a la calidad educativa que se desarrolla en sus centros asociados? Es decir, el trabajo de investigación tratará de conocer y estudiar la realidad de la enseñanza a distancia en esta Universidad con el objetivo de mejorar aspectos sustanciales de la misma. 
En líneas generales, se trata de hacer una investigación de tipo cuantitativo, haciendo uso de una metodología descriptiva y correlacional.

Para ello y sobre la base de resultados que obtengamos de la presente investigación, esperamos que se puedan tomar decisiones a futuro, tales como hacer propuestas de mejora que se puedan derivar del uso de las nuevas tecnologías en los procesos de aprendizaje y formación de los estudiantes de la UNED.

\section{CUESTIONARIO PROPUESTO A ESTUDIANTES DE LA UNED}

El cuestionario tuvo por objetivo poder contrastar sus opiniones y conocer la disposición de éstos hacia las nuevas tecnologías.

Por ello se les indicó que, si se decidían a responderlo, meditasen sus respuestas contestando a las mismas con entera libertad ya que era totalmente anónimo, sin otro sentido que conocer más de cerca los problemas tecnológicos, si los hubiera, que se les plantease durante su proceso de formación, para poder ofrecer respuestas que permiten, dentro de lo posible, solucionar dichos problemas.

Se les indicó así mismo que en las ocho preguntas ofrecidas en el cuestionario, había dos tipos distintos; las preguntas de varias opciones, en las que debían rodear con un círculo la que mejor reflejara su opinión, y las de escalas de valoración (de 1 a 5) en las que deberían rodear con un círculo aquella puntuación que mejor definiera su opinión, teniendo en cuenta que el número 1 está asociado al valor más bajo y que el número 5 al valor más alto. Si en ambos casos se quisiera rectificar la respuesta, no habría más que tachar la opción errónea y señalar la adecuada.

En el anexo 1, figuran las preguntas propuestas en el cuestionario mencionado, con el objetivo de conocer la opinión de los estudiantes encuestados, así como obtener unas conclusiones finales que nos permitan poder desarrollar un trabajo similar con profesionales de la educación como son, los profesores tutores y profesores de la sede central de la UNED.

Después de haber obtenido información de García Llamas y otros (2003), se recurrió al manejo del paquete estadístico SPSS para mostrar los resultados obtenidos. No obstante, y con el fin de que fuese altamente fiable el estudio realizado, procedimos anteriormente, a analizar la validez y contenido de dicho cuestionario. 


\section{ANÁLISIS DE LA FIABILIDAD Y VALIDEZ DEL CUESTIONARIO}

Dado que se ha empleado un instrumento de recogida de datos como es el cuestionario, quisimos ver si éste era fiable y válido es decir, interesaba contrastar si la variación observada en las puntuaciones alcanzadas por los encuestados lo era por la característica que se estaba analizando o, por el contrario, podía estar motivada por otras influencias.

A este respecto debemos indicar que dentro de las Teorías Clásicas de los Tests, (TCT); el método de consistencia interna es el camino más habitual para estimar la fiabilidad de las pruebas, escalas o tests, cuando se utilizan conjuntos de ítems que se espera midan el mismo atributo o campo de contenido, dónde la consistencia interna es el grado común que tienen todos los ítems, siendo dentro de esta categoría el coeficiente alfa de Cronbach $^{8}$ (1951), sin duda, el más ampliamente utilizado por los investigadores.

La principal ventaja de este método es que requiere sólo una administración de la prueba, además de estar disponible, como opción de análisis, en los principales y más conocidos programas estadísticos, como es el SPSS.

Este estadístico, toma valores entre o y 1 y aunque también puede tomar valores negativos, lo cuál indicaría que en la escala hay ítems que miden lo opuesto al resto, ello no sería correcto ya que viola los supuestos del modelo de fiabilidad, debido a una covarianza promedio entre los elementos negativos.

En este sentido, Grady y Wallston (1988) nos indican que "en general, un valor de 0.60 es aceptable para la confiabilidad medida con el alfa de Cronbach": Por su parte, Nunnaly (1978) apunta que: "el alfa de Cronbach, en pruebas de consistencia interna, es bueno por encima de 0.7". En este misma línea, se pronuncian George y Mallery (1995) 'el alfa de Cronbach... entre 0.7 y 0.8 haría referencia a un nivel aceptable; en el intervalo o.8-0.9 se podría calificar de un buen nivel, y si tomara valores superiores a 0.9 , seria excelente".

Por todo ello podemos afirmar que cuanto más se acerque el coeficiente a la unidad, mayor será la consistencia interna de los indicadores de la escala evaluada $\mathrm{y}$, aunque, como se ha visto, no existe un acuerdo generalizado sobre cuál es el límite inferior que indica cuándo una escala puede ser considerada fiable o no, es, aproximadamente, a partir de $\alpha=0.7$, cuando el valor es estadísticamente aceptable para dar fiabilidad a la escala evaluada. 
En nuestro estudio de fiabilidad del cuestionario, se ha realizado el cálculo del alfa de Cronbach que como puede observarse arroja un $\alpha=0.818$ valor que, según nos indican George y Mallery (1995), es un buen nivel.

\section{Estadísticos de fiabilidad}

\begin{tabular}{|c|c|}
\hline Alfa de Cronbach & N de elementos \\
\hline, 818 & 20 \\
\hline
\end{tabular}

Una vez finalizado el estudio de fiabilidad del cuestionario y a la vista de los resultados obtenidos, podemos afirmar que el cuestionario del anexo I, respecto a los ítems que figuran en el mismo, es totalmente fiable dado el valor alcanzado por el alfa de Cronbach.

En cuanto a la eficacia del mismo, se ha recurrido a la validez del contenido, por creer que es el procedimiento más adecuado para este tipo de trabajo de investigación. La determinación de la validez del contenido, se ha conseguido mediante el juicio emitido por una serie de personas expertas en el trabajo, afirmando todos ellos la correcta confección del cuestionario.

Dichos expertos, deberían reunir al menos dos características:

- Formación científica en educación a distancia, educación de adultos, tecnologías y metodología en la educación.

- Práctica docente en esta modalidad de enseñanza.

\section{ESTUDIO DESCRIPTIVO-CUANTITATIVO}

Los datos más significativos aportados por la muestra se recogen a continuación, teniendo en cuenta aquellas variables de categorización más importantes del estudio, las cuáles, figuran a continuación.

\section{Carrera}

El cuestionario se pasó a estudiantes de las Facultades de: Educación, Derecho, Administración y Dirección de Empresas (ADE)-Económicas, Geografía e Historia, Filosofía-Antropología, Ingeniería y Ciencias, figurando a continuación la distribución de estudiantes por carreras. 


\begin{tabular}{|l|l|l|l|l|}
\hline \multicolumn{2}{|c|}{} & Frecuencia & Porcentaje & $\begin{array}{c}\text { Porcentaje } \\
\text { acumulado }\end{array}$ \\
\hline \multirow{4}{*}{ Válidos } & Educación & 421 & 28,5 & 28,5 \\
\cline { 2 - 5 } & Derecho & 228 & 15,5 & 44,0 \\
\cline { 2 - 5 } & Ade-Económicas & 190 & 12,9 & 56,9 \\
\cline { 2 - 5 } & Geografía-Historia & 181 & 12,3 & 69,2 \\
\cline { 2 - 6 } & Filosofía-Antropología & 158 & 10,7 & 79,9 \\
\cline { 2 - 6 } & I. Industrial & 75 & 5,1 & 84,9 \\
\hline \multirow{6}{*}{} & I. Informático & 119 & 8,1 & 93,0 \\
\cline { 2 - 6 } & Ciencias & 103 & 7,0 & 100,0 \\
\cline { 2 - 5 } & Total & 1475 & 100,0 & \\
\hline
\end{tabular}

Cuadro 1. Carreras

En la representación gráfica de sectores que figura a continuación observamos que casi la tercera parte de los estudiantes encuestados están realizando los estudios de Educación (28,5\%), seguida por los alumnos de Derecho que con un porcentaje del $(15,5 \%)$, indica cuáles son las dos carreras más representadas en la muestra, mientras que las carreras de la rama Tecnológicas- Científicas, Ingenierías y Ciencias, con un porcentaje del $(13,2 \%)$ para las primeras y del (7\%) para las segundas, figuran entre las que menos porcentaje aportan a la muestra.

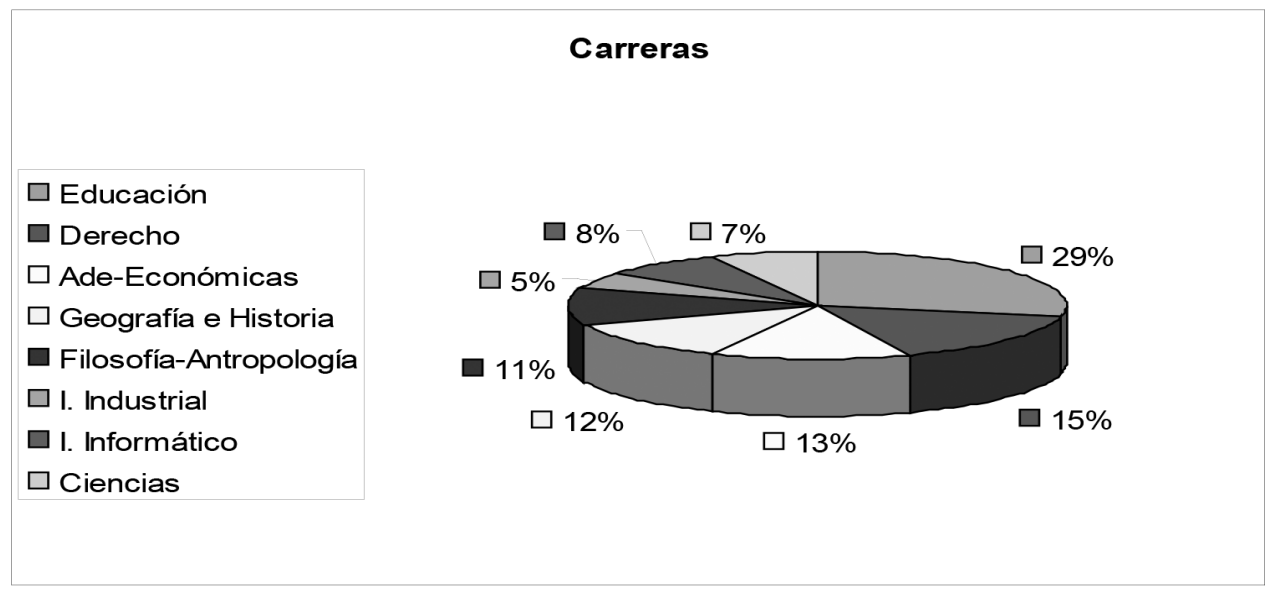

Gráfico 1. 


\section{Curso}

\begin{tabular}{|l|l|l|l|l|}
\hline \multicolumn{2}{|l|}{} & Frecuencia & Porcentaje & $\begin{array}{c}\text { Porcentaje } \\
\text { acumulado }\end{array}$ \\
\hline Válidos & $1^{\mathrm{o}}$ & 515 & 34,9 & 34,9 \\
\hline & $2^{\mathrm{O}}$ & 357 & 24,2 & 59,1 \\
\hline & $3^{\mathrm{O}}$ & 293 & 19,9 & 79,0 \\
\hline & $4^{\mathrm{O}}$ & 172 & 11,7 & 90,6 \\
\hline & $5^{\mathrm{O}}$ & 138 & 9,4 & 100,0 \\
\hline & Total & 1475 & 100,0 & \\
\hline
\end{tabular}

Cuadro 2. Curso

Como puede observarse más de la tercera parte de los cuestionarios indican que los estudiantes se encuentran en el primer curso de carrera $(34,9 \%)$, siendo casi el $60 \%$ los estudiantes que realizan los dos primeros cursos (59,1\%), el porcentaje desciende conforme avanza el curso en el que están matriculados, tan sólo el $(9,4 \%)$ de los estudiantes están matriculados en el último curso de carrera.

El diagrama de frecuencias nos confirma, mediante una rápida visualización, las observaciones realizadas con anterioridad: 872 de los 1475 estudiantes están matriculados entre los dos primeros cursos de carrera. 


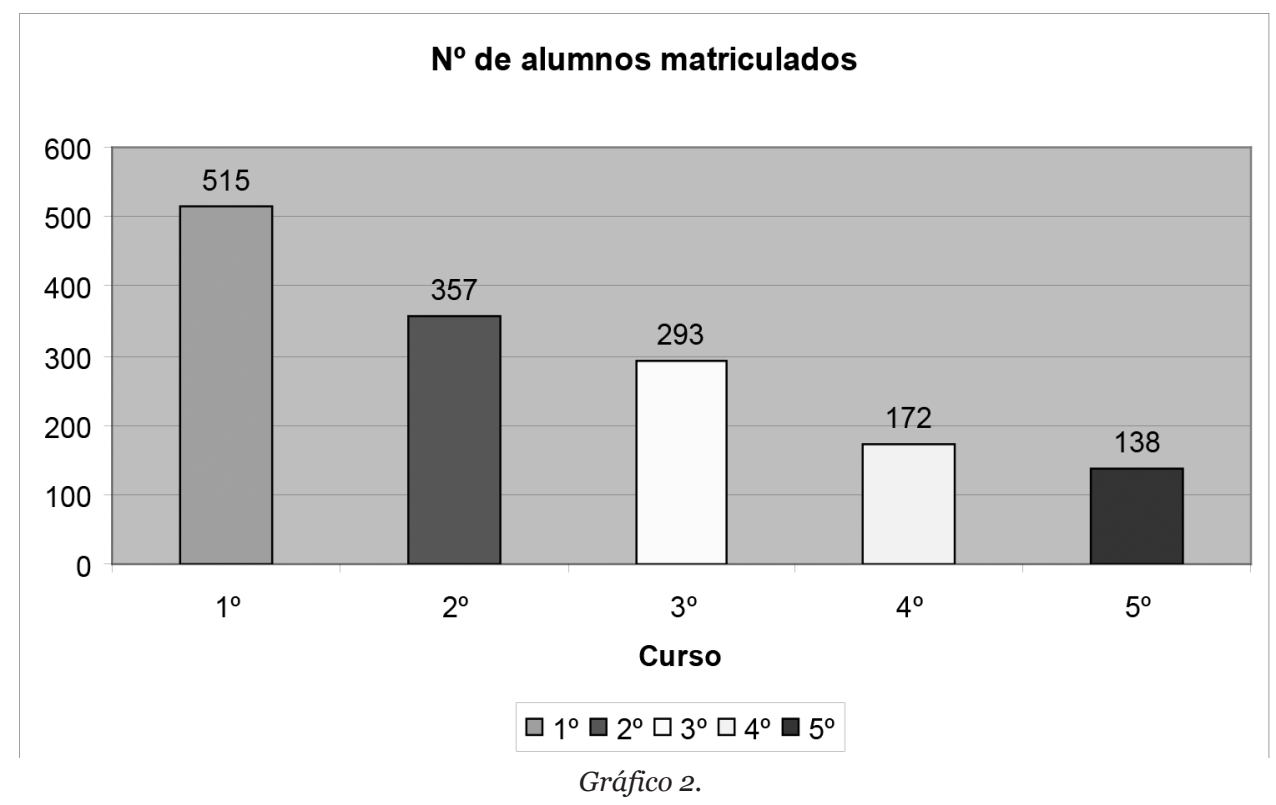

Sexo

Aunque efectivamente hay una mayor presencia femenina $(51,9 \%)$ en los cuestionarios recibidos, sin embargo los porcentajes obtenidos son bastantes similares ya que el restante $(48,1 \%)$ lo respondieron varones, por lo que podemos indicar que en la realización de nuestro estudio está muy compensada la opinión de ambos sexos.

\begin{tabular}{|l|l|l|l|l|}
\hline \multicolumn{2}{|c|}{} & Frecuencia & Porcentaje & \multicolumn{1}{|c|}{$\begin{array}{c}\text { Porcentaje } \\
\text { acumulado }\end{array}$} \\
\hline \multirow{3}{*}{ Válidos } & Hombre & 709 & 48,1 & 48,1 \\
\cline { 2 - 5 } & Mujer & 766 & 51,9 & 100,0 \\
\cline { 2 - 5 } & Total & 1475 & 100,0 & \\
\hline
\end{tabular}

Cuadro 3. Sexo

\section{Nivel de conocimientos informáticos que tiene}

La frecuencia de estudiantes que respondieron a dicho ítem fue muy elevada ya que, como indican los estadísticos, fueron 1471 estudiantes los que enviaron el mismo. 
A la vista de los resultados obtenidos, en general, podemos afirmar que los conocimientos informáticos de los estudiantes de la UNED son aceptables, siendo la opción "regulares" la que alcanza el porcentaje más alto (36,3\%) respecto de las otras opciones ofrecidas en el cuestionario. Dicho porcentaje válido aumenta hasta el (66,5\%), las dos terceras partes de las respuestas ofrecidas por los estudiantes, si además incluimos la opción "bastantes", en el análisis del ítem.

El histograma que se presenta a continuación, nos muestra cómo los datos obtenidos en este ítem se ajustan a la normalidad pues desde la opción "pocos" asciende el porcentaje hasta "regulares", descendiendo después hasta "muchos", pasando por el punto medio de la base superior de los rectángulos.

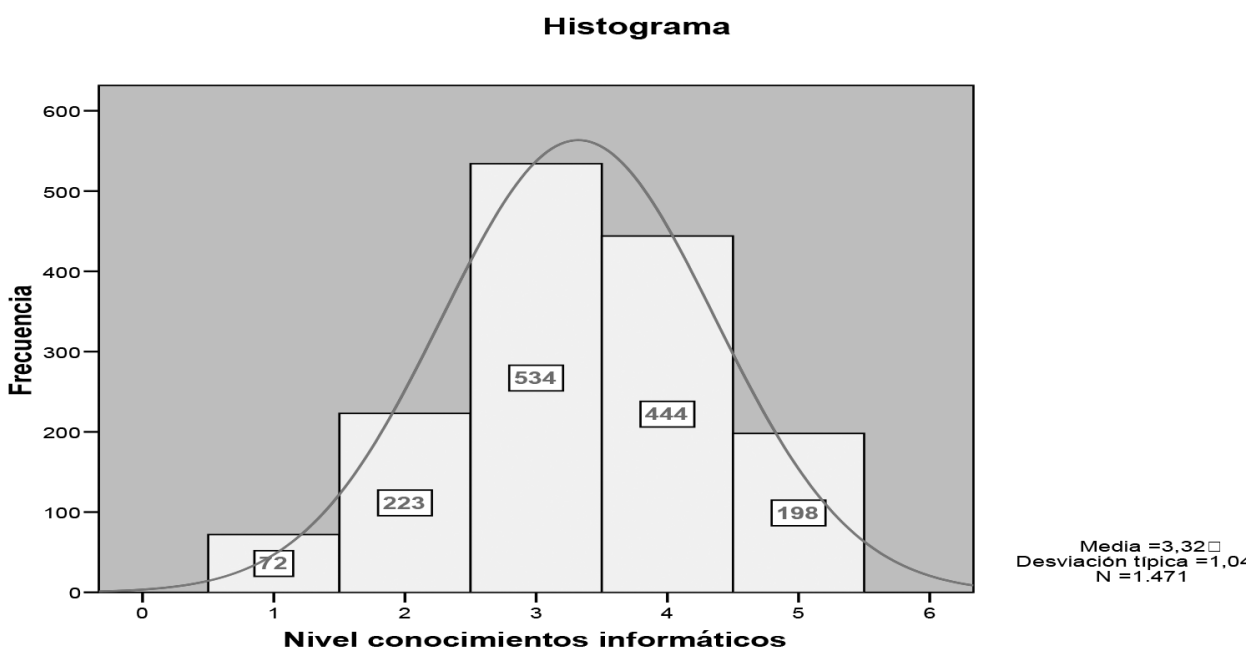

Gráfico 3

\section{Tipo de ordenador personal}

Los datos aportados por el paquete estadístico SPSS respecto a la opción del ítem, "Tipo de ordenador personal" que tienen los estudiantes de la UNED, indican que más de la mitad de ellos, 776 estudiantes, afirmaron tener un ordenador de sobremesa mientras que otros 401 estudiantes indicaron tener un ordenador portátil. Entre ambas opciones suman el $(79,8 \%)$ del porcentaje válido de estudiantes (1177), que indicaron tener al menos un tipo de ordenador. Así mismo consideramos también elevado el número de estudiantes que afirmaron tener ambos tipos de ordenador ya que 224 de ellos, el (15,2\%), nos lo confirmaron a través de sus respuestas. 
Por último señalar cómo 73 estudiantes, que corresponde a un porcentaje válido del (5\%), afirmaron no tener ningún tipo de ordenador. Aunque no es un porcentaje elevado, pensamos que en los tiempos de "Tecnologías de la Información y Comunicación, $T I C^{\circ}$ ' actuales, no debería haber ningún estudiante, y más en una Universidad a Distancia, sin este tipo de comunicación.

La gráfica de barras conoidales, nos muestra las frecuencias de cada una de las opciones que figuran en el ítem que acabamos de analizar donde sobresale la opción de tener un ordenador personal de "Sobremesa".

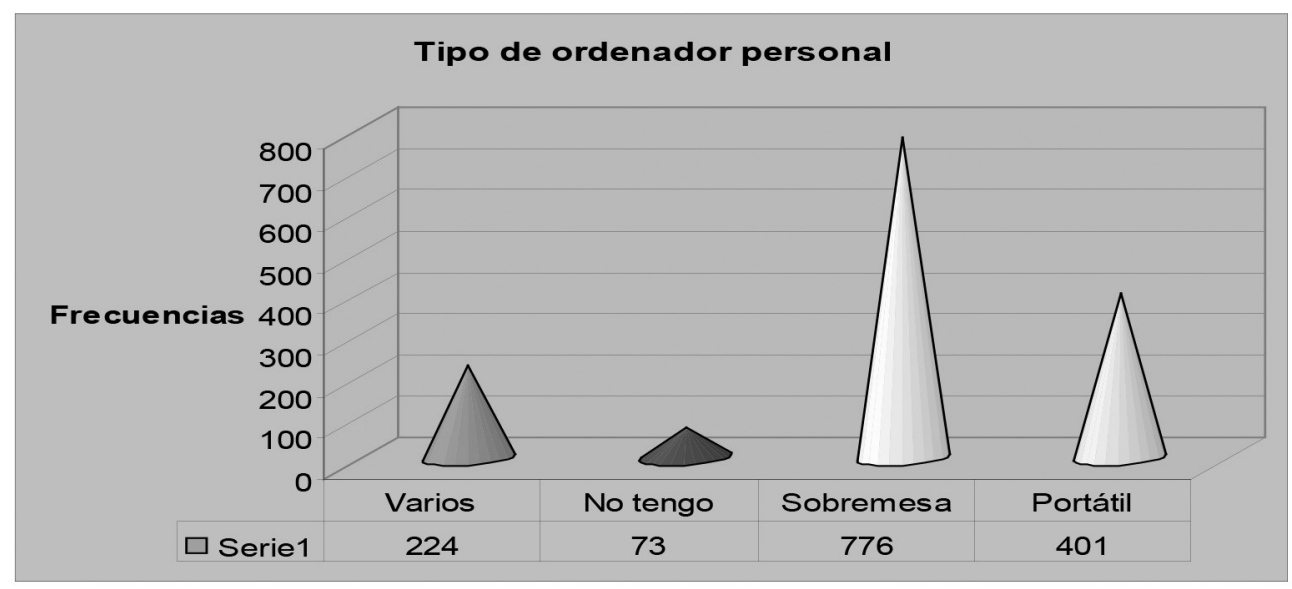

Gráfico 4.

\section{Conexión a Internet disponible en su domicilio}

Más de la mitad de los estudiantes (53,3\%), afirmaron tener en su domicilio una "Conexión banda ancha/alta velocidad", como tipo de conexión, disponible en su domicilio, a Internet.

$\mathrm{Al}$ igual que indicamos en el ítem anterior y conforme los resultados que figuran en el siguiente cuadro, nos parece muy elevado que el $(17,6 \%)$ de los estudiantes afirme que no dispone de ningún tipo de conexión a Internet en su domicilio, máxime cursando estudios en una universidad a distancia y en un mundo globalizado, dónde Internet es fundamental para este tipo de estudios.

En la gráfica de sectores, que se muestra a continuación, figuran las tres opciones ofrecidas a los estudiantes en el cuestionario, dónde "conexión banda ancha/alta velocidad" sobresale sobre las otras dos opciones del ítem. 


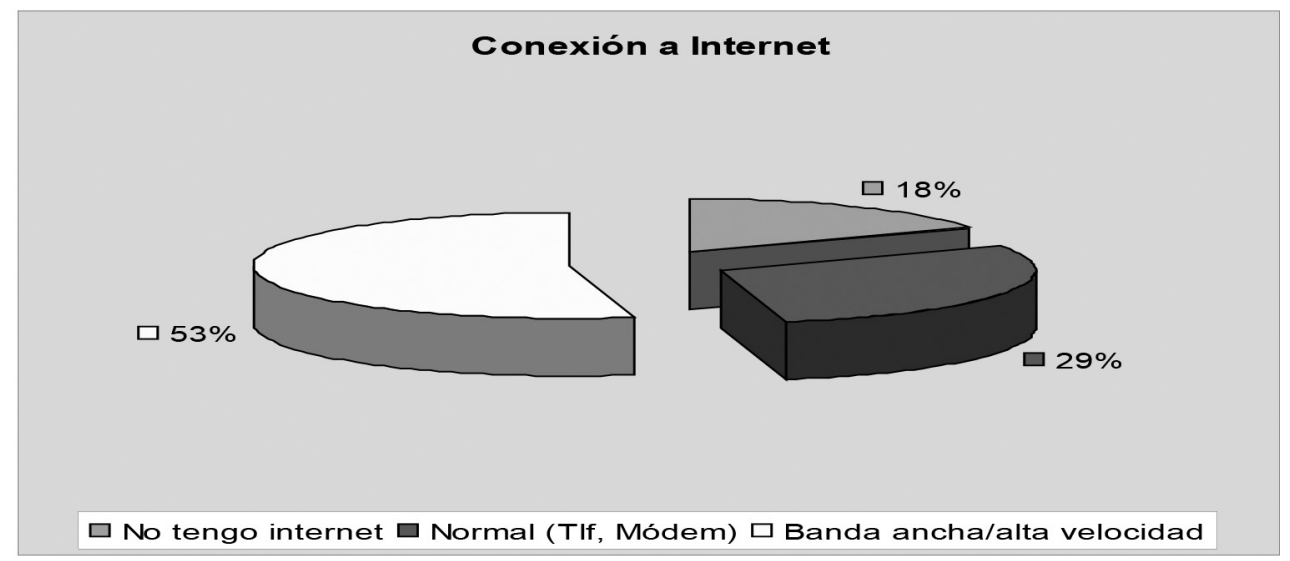

Gráfico 5 .

\section{Disposición de uso de nuevas tecnologías, Internet, en su Centro Asociado}

De entre las dos opciones que se ofrecían en el ítem, el (71,3\%) de los estudiantes afirma poder disponer del uso de Internet en su Centro Asociado, mientras que el resto $(28,7 \%)$, indicó lo contrario.

Con respecto a los datos obtenidos, hemos de indicar que mayoritariamente los estudiantes de la UNED pueden disponer de esta tecnología en su Centro Asociado, lo cual es lógico si tenemos en cuenta las consideraciones realizadas en los ítems anteriores respecto a las TIC, tan necesarias en los tiempos actuales e imprescindibles en la enseñanza a distancia.

La gráfica sectorial nos muestra el porcentaje de alumnos que disponen de uso de Internet en su Centro Asociado. 


\section{Disposición uso Internet en C.A}

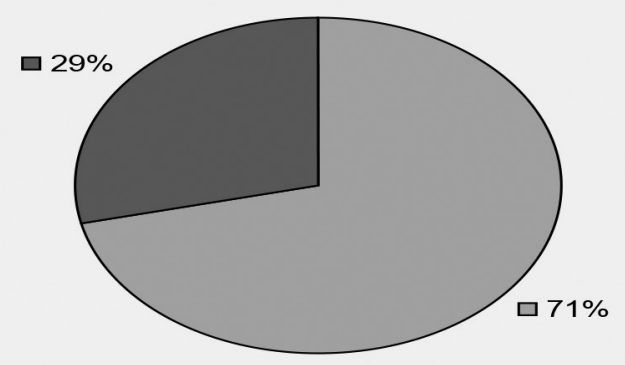

$\square$ sí $\square$ no

Gráfico 6.

\section{Nivel de participación en foros}

De las opciones que se ofrecieron en el cuestionario, "Varias", que incluye cuestionarios que respondieron al menos a dos de las opciones individuales ofrecidas, fue la que obtuvo un mayor porcentaje de estudiantes, al ser el (43,1\%) de ellos los que así lo manifestaron. Entre las opciones individuales, fue "Como lector", la que con un $(25,3 \%)$ de cuestionarios registró el mayor porcentaje válido entre las mismas.

Así mismo un porcentaje importante de estudiantes, el (19,8\%), se ha manifestado en el sentido de que nunca ha participado en los foros.

La gráfica que visualizamos nos ofrece las frecuencias absolutas de cada una de las opciones incluidas en el cuestionario. 


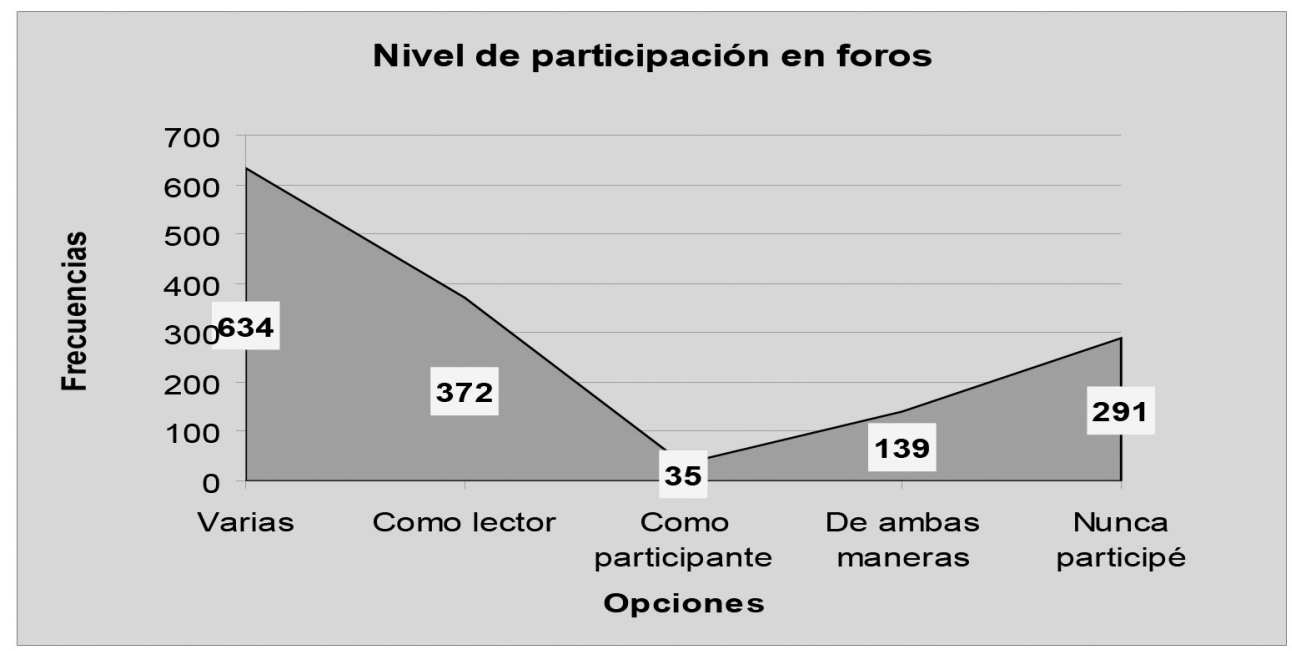

Gráfico 7.

Valoración, si los conoce, de los "TAR". Tutores de apoyo en Red

Como se puede observar, existe un alto porcentaje de estudiantes que afirma no conocer la figura de los "TAR" (63,2\%). El porcentaje es lo suficientemente elevado como para indicar que los estudiantes de la UNED, no utilizan esta figura como apoyo en sus estudios, figura que creemos les sería de gran ayuda; como complemento a sus tutorías, para los que asisten a ellas y/o como apoyo a las dudas que les puedan ir surgiendo, para los que no pueden asistir a las mismas. Los estudiantes que afirmaron conocer la figura del "TAR", "bastante" o "mucho", es de tan sólo el (20,4\%).

La gráfica que aparece a continuación, nos indica claramente las observaciones que se han realizado sobre éste ítem. 


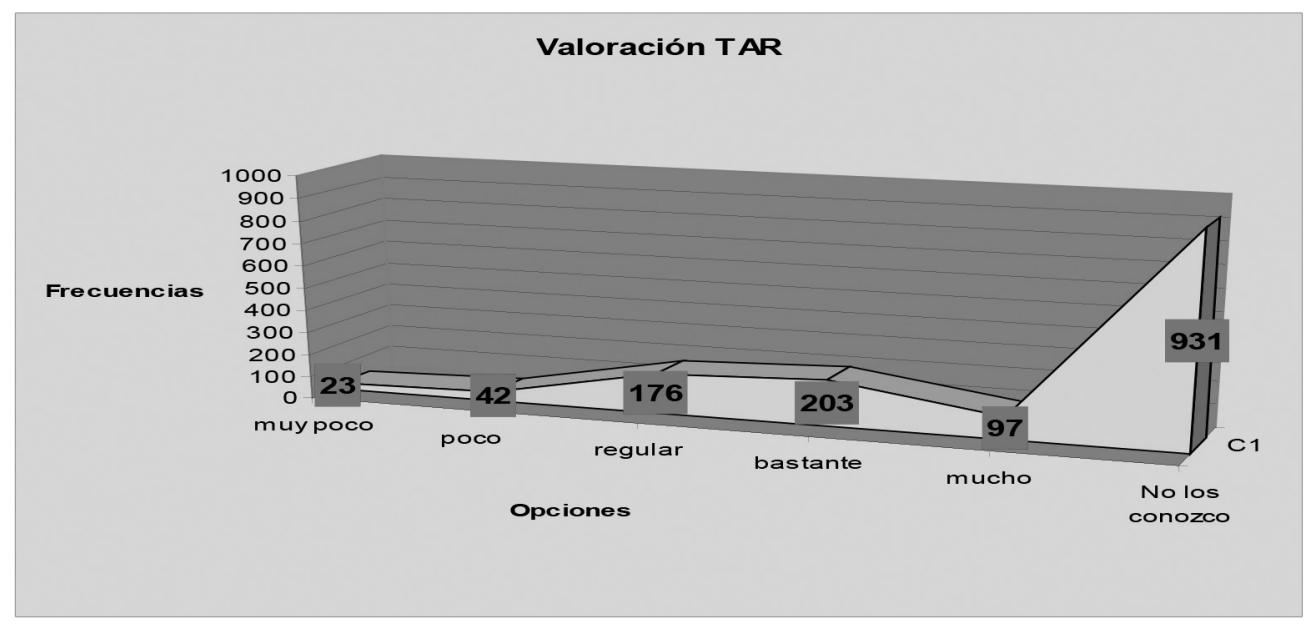

Gráfico 8.

\section{Existen portales, o páginas Web, de sus asignaturas en la Red}

Los estadísticos que figuran a continuación, nos indican que el $(31,9 \%)$ de los estudiantes afirma que existen portales, o páginas Web, de todas sus asignaturas en la Red, lo cuál muestra cómo la UNED, como universidad a distancia que es, en general, tiene portales o páginas Web, de prácticamente la totalidad de las asignaturas.

En la gráfica sectorial desgajada que figura a continuación, podemos observar los diferentes porcentajes válidos de cada una de las opciones sobre la existencia, o no, de portales, o páginas Web, de las asignaturas de los estudiantes de la UNED. 


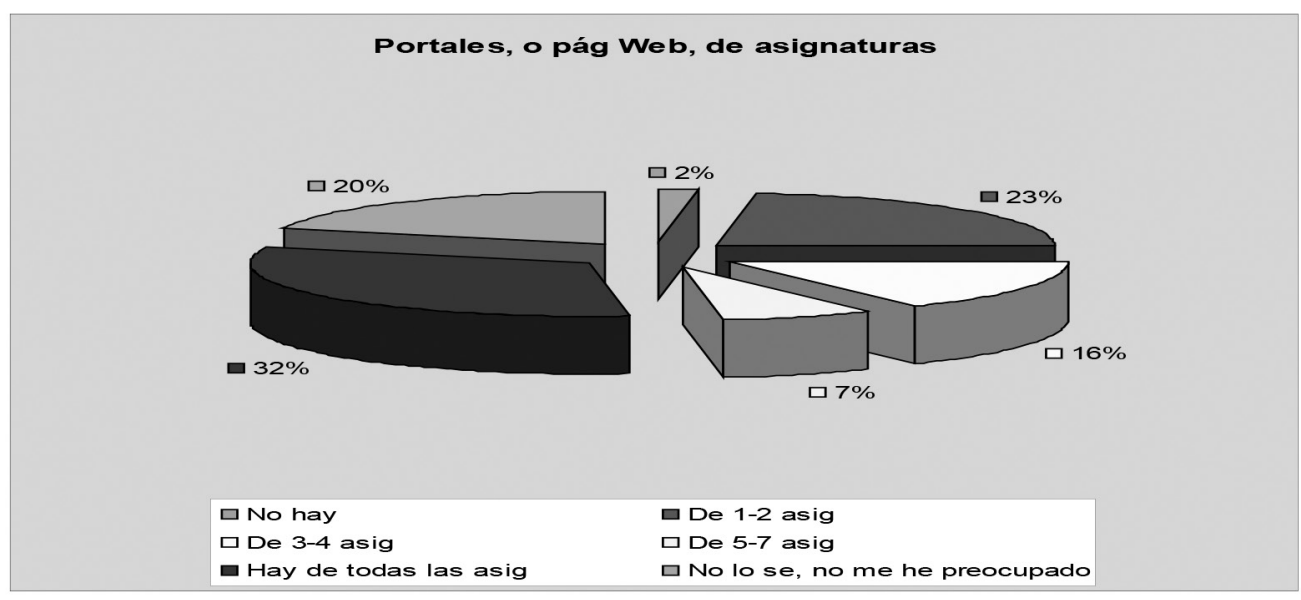

Gráfico 9.

Debemos recordar que en el Espacio Europeo de Educación Superior (EEES), las páginas Web de las asignaturas de grado son obligatorias.

\section{Hace uso de las nuevas tecnologías en la preparación de sus asignaturas}

Es el último de los ítems del cuestionario propuesto a los estudiantes dónde un porcentaje válido muy elevado de ellos (62,8\%) afirmó aplicar las nuevas tecnologías en la preparación de sus asignaturas. En concreto son 917 estudiantes, de los 1460 que respondieron al ítem.

El siguiente histograma de frecuencias, con la curva normal incluida, nos muestra como efectivamente la opción del ítem, "Las aplico siempre que me es necesario", destaca sobre todas las demás opciones del mismo. 


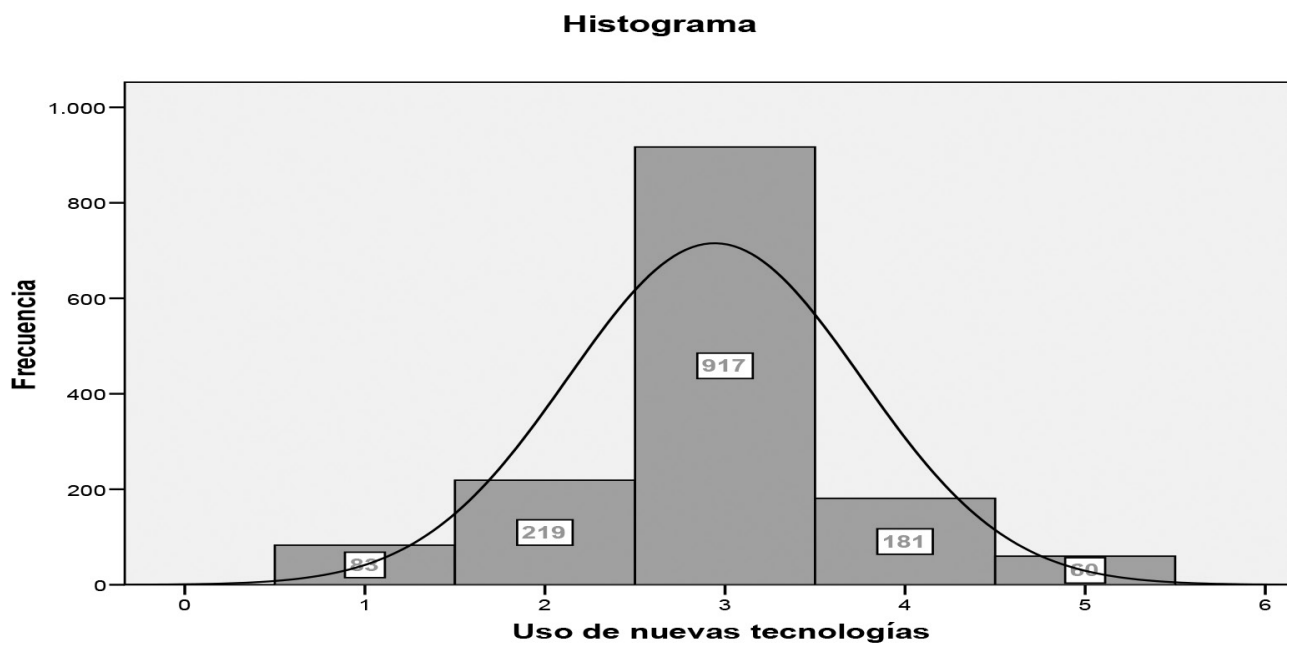

Gráfico 10.

\section{ESTUDIO COMPARATIVO DE LOS DATOS}

El análisis comparativo de los datos, se ha realizado entre la variable carrera y las variables del cuestionario de los estudiantes.

En un segundo momento, realizamos el análisis comparativo entre la "Carrera", y las variables de "Disposición de nuevas tecnologías", "Nivel de conocimientos informáticos que tiene" y "Valoración, si los conoce, de los TAR ", ofreciéndonos la tabla ANOVA: promedios, valor de F, y nivel de significación, de las variables cuantitativas de dicho bloque; con el objetivo de conocer si existen diferencias significativas entre ellas.

\section{Carreras}

\begin{tabular}{|c|c|c|c|c|c|c|c|c|c|c|}
\hline Ítem & 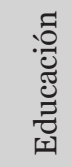 & 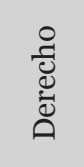 & 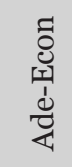 & $\begin{array}{l}\frac{\pi}{11} \\
\vdots \\
0 \\
0\end{array}$ & 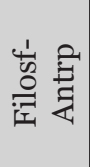 & $\underset{\Xi}{\Xi}$ & 岂 & 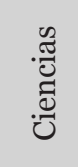 & I & $\frac{.00}{\sqrt{2}}$ \\
\hline $\begin{array}{l}\text { Nivel } \\
\text { conocimientos } \\
\text { informáticos }\end{array}$ & 3,17 & 3,10 & 3,57 & 3,13 & 3,18 & 3,45 & 4,00 & 3,67 & 15,598 & , оoo \\
\hline Valoración TAR & 5,03 & 5,29 & 5,07 & 5,31 & 5,12 & 5,03 & 4,93 & 4,97 & 1,999 & ,052 \\
\hline
\end{tabular}

Cuadro 4. ANOVA 
La variable "Nivel de conocimientos informáticos" es la única que presenta diferencias significativas, al nivel de confianza del 95\%, y una F=15,598, elevada, por lo que procedemos a su análisis.

A la vista de los resultados obtenidos, son los estudiantes de Ingeniería Informática (4 puntos) y los de Ciencias $(3,67)$ los que indican tener mayor nivel de conocimientos informáticos que el resto de sus compañeros; mientras que los de Geografía e Historia y Derecho, con 3,13 y 3,1 puntos respectivamente, son los que menos nivel de estos conocimientos poseen.

Realizada a continuación la prueba de homogeneidad de varianzas de Levene, y como puede observarse en el cuadro siguiente, ambas cumplen la igualdad de varianzas, por lo que realizaremos a continuación la prueba de robustez de igualdad de medias de Welch, para asegurarnos si efectivamente dichas variables presentan diferencias significativas entre sus medias.

\begin{tabular}{|l|c|c|c|c|}
\hline & $\begin{array}{c}\text { Estadìstico } \\
\text { de levene }\end{array}$ & gl1 & gl2 & Sig. \\
\hline Nivel conocimientos informáticos & 2,423 & 7 & 1463 & 0,018 \\
Valoracion TAR & 3,032 & 7 & 1464 & 0,004 \\
\hline
\end{tabular}

Cuadro 5. Prueba de homogenidad de varianzas

\begin{tabular}{|c|c|c|c|c|c|}
\hline & & Estadìstico $^{a}$ & gl1 & gl2 & Sig. \\
\hline $\begin{array}{l}\text { Nivel conocimientos } \\
\text { informáticos }\end{array}$ & Welch & 16,370 & 7 & 457,885 & 0,000 \\
\hline Valoracion TAR & Welch & 2,037 & 7 & 455,049 & 0,049 \\
\hline
\end{tabular}

Cuadro 6. Pruebas robustas de igualdad de las medidas

Por ser la significación de ambas variables menores que 0,05, realizamos a continuación, sobre las mismas, la prueba de comparaciones múltiples, que no asume igualdad de varianzas, de Tamhane.

Dicha prueba sólo ofreció diferencia significativas entre las medias de la variable "Nivel de conocimientos informáticos", lo cuál parece lógico, dada la proximidad a $(0,05)$ obtenida por la significación de la otra variable, "Valoración, si los conoce, de los TAR" de $(0,49)$. 


\section{Tamhane}

\begin{tabular}{|c|c|c|c|c|c|c|c|}
\hline \multirow{2}{*}{$\begin{array}{c}\text { Variable } \\
\text { dependiente }\end{array}$} & \multirow{2}{*}{ (I) Carrera } & \multirow{2}{*}{ (J) Carrera } & \multirow{2}{*}{$\begin{array}{c}\text { Diferencia de } \\
\text { medias (I-J) } \\
\text { Límite } \\
\text { inferior }\end{array}$} & \multirow{2}{*}{$\begin{array}{l}\text { Error } \\
\text { típico } \\
\text { Límite } \\
\text { superior }\end{array}$} & \multirow{2}{*}{$\begin{array}{l}\text { Sig. } \\
\text { Límite } \\
\text { inferior }\end{array}$} & \multicolumn{2}{|c|}{$\begin{array}{c}\text { Intervalo de } \\
\text { confianza al } 95 \%\end{array}$} \\
\hline & & & & & & $\begin{array}{c}\text { Límite } \\
\text { superior }\end{array}$ & $\begin{array}{l}\text { Límite } \\
\text { inferior }\end{array}$ \\
\hline \multirow{17}{*}{$\begin{array}{l}\text { Nivel } \\
\text { conocimientos } \\
\text { informáticos }\end{array}$} & \multirow{7}{*}{$\begin{array}{l}\text { Ade- } \\
\text { Económicas }\end{array}$} & Educación &, $395\left(^{*}\right)$ & ,082 & ,000 & ,14 & ,65 \\
\hline & & Derecho &, $469\left(^{*}\right)$ & 101 & , ooo & ,15 & ,78 \\
\hline & & Geografía e &, $439\left(^{*}\right)$ & 100 & , 000 & 13 & ,75 \\
\hline & & $\begin{array}{l}\text { Historia } \\
\text { Filosofía- }\end{array}$ & 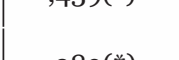 & & & & \\
\hline & & Antropología &, $389\left(^{*}\right)$ & 109 & ,012 & ,05 & ,73 \\
\hline & & I. Informática &,$- 434\left(^{*}\right)$ & ,110 & ,o03 &,- 78 &,- 09 \\
\hline & & Educación &, $829(*)$ & ,100 & , ooo &, 51 & 1,14 \\
\hline & \multirow{7}{*}{$\begin{array}{l}\text { I. } \\
\text { Informática }\end{array}$} & Derecho &, $903\left(^{*}\right)$ & ,115 & ,ooo &, 54 & 1,27 \\
\hline & & $\begin{array}{l}\text { Ade- } \\
\text { Económicas }\end{array}$ &, $434\left(^{*}\right)$ & 110 & ,003 & ,09 &, 78 \\
\hline & & Geografía e & & & & & \\
\hline & & Historia &, $873\left(^{*}\right)$ & 114 & , &, 51 & 1,23 \\
\hline & & $\begin{array}{l}\text { Filosofía- } \\
\text { Antropología }\end{array}$ &, $823(*)$ & 123 & , 000 & 44 & 1,21 \\
\hline & & I. Industrial &, $554\left(^{*}\right)$ & ,149 & ,008 & ,08 & 1,03 \\
\hline & & Educación & $499\left(^{*}\right)$ & 108 & , ooo & 16 & ,84 \\
\hline & \multirow{3}{*}{ Ciencias } & Derecho &, $573\left(^{*}\right)$ & ,123 & , & ,19 & ,96 \\
\hline & & $\begin{array}{l}\text { Geografía e } \\
\text { Historia }\end{array}$ &, $543(*)$ & 122 & ,OOO & ,16 & ,93 \\
\hline & & $\begin{array}{l}\text { Filosofía- } \\
\text { Antropología }\end{array}$ & ,493(*) & ,130 & ,005 & ,08 & ,90 \\
\hline
\end{tabular}

Cuadro 7. Comparaciones múltiples

* La diferencia de medias es significativa al nivel .05.

Como puede observarse, la carrera que produce diferencias fundamentales con las medias de las demás carreras, excepto Ciencias, es Ingeniería Informática, siendo dicha diferencia más acusada con la carrera de Derecho, $(0,903)$. 


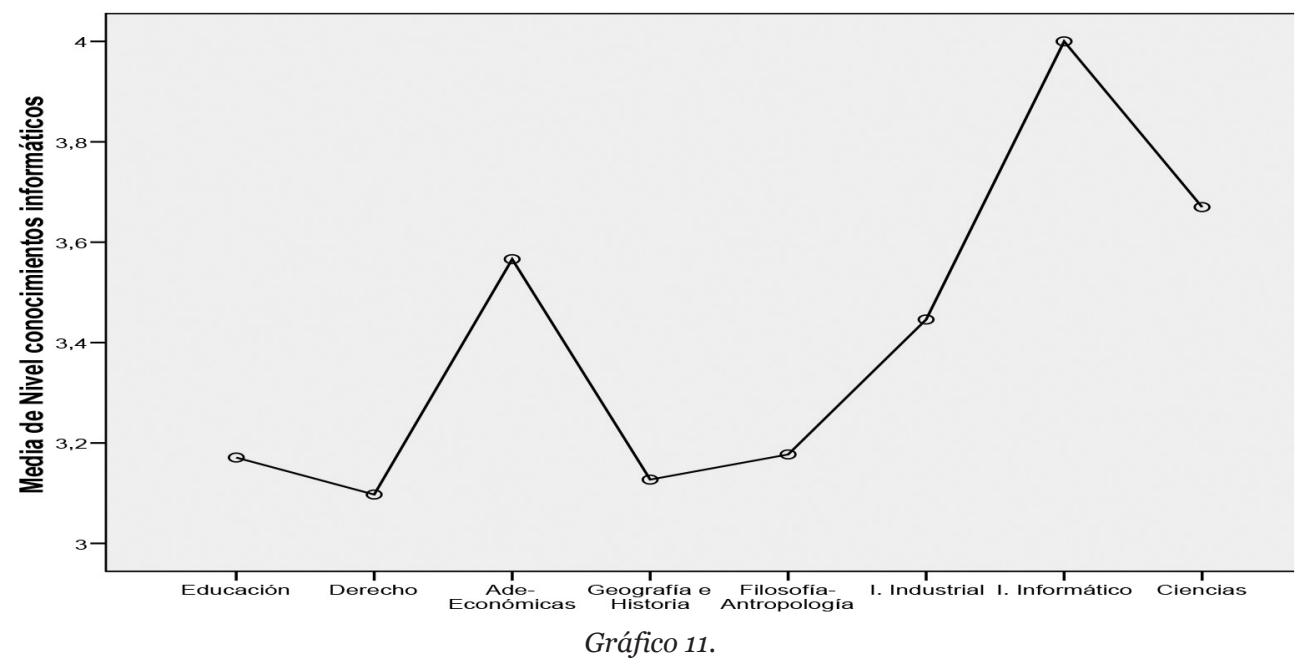

La gráfica de medias anterior pone de manifiesto la afirmación realizada, dónde además de con la carrera de Derecho, las medias producidas entre la Ingeniería de Informática y las carreras de Educación, Geografía e Historia y FilosofíaAntropología, también son bastante significativas.

Tiene lógica lo comentado anteriormente ya que es de suponer que los estudiantes de Ingeniería y Ciencias, y en concreto los Informáticos, tienen un nivel superior al resto de estudiantes, unos por utilizarla con mayor asiduidad en sus estudios, y los otros, por ser materia específica de sus estudios.

\section{ANÁLISIS}

Una vez recibidos los 1627 cuestionarios de los estudiantes, de los que consideramos válidos 1475, al ser los enviados por estudiantes de las carreras objeto de estudio, y antes de analizar los mismos, establecimos unos filtros que minimizasen al máximo los posibles errores cometidos; tanto en las respuestas dadas por los estudiantes, algunas de ellas se contradecían, como por nuestra parte a la hora de introducir dichos datos.

Concluida esta labor, introdujimos éstos en un fichero de datos con el objetivo de tratar los mismos con el programa estadístico SPSS.

Una vez que toda la muestra aceptada estuvo bastante depurada, comenzó el análisis global de los datos recogidos en los diferentes centros asociados de la 
UNED, 39, realizando para ello, un análisis de tipo descriptivo sobre las: frecuencias, porcentajes, medias, desviaciones típicas y representaciones gráficas, con el objetivo de tener una visión rápida sobre el comportamiento y situación de la totalidad de la muestra la cuál, incluía cuestionarios de las 17 Comunidades Autónomas.

A la vista de los resultados obtenidos, creemos que el uso de las Tecnologías de la Información y Comunicación son fundamentales, tanto a nivel educativo como organizativo en las instituciones educativas.

Siendo conciente de ello, la UNED ha dispuesto una estructura tecnológica en su organización que le permite mantenerse como una Universidad pionera en la docencia a distancia facilitando el "encuentro" entre los distintos estamentos educativos al proporcionar las herramientas necesarias para que la distancia no sea obstáculo y aproximar así tanto estudiantes como docentes mediante el uso de esas herramientas tecnológicas.

En nuestro análisis, se han analizado las principales herramientas tecnológicas puestas por la Universidad al servicio de sus estudiantes así como su funcionalidad, adaptándose las mismas tanto a las Tecnologías de la Comunicación como a los intereses de los estudiantes.

De dicho análisis, debemos destacar cómo los conocimientos informáticos de los estudiantes de la UNED, respecto a la aplicación de las nuevas tecnologías, son aceptables, con 3.32 puntos de media sobre 2.5, afirmando tener ordenador de sobremesa el (52.6\%), lo que posibilita el uso de Internet, mediante la conexión banda ancha de alta velocidad, en su domicilio al (53.3\%) de los estudiantes. Además, para ese porcentaje de estudiantes que indicaron no tener Internet en su domicilio (17.6\%), los centros asociados lo ponen a su disposición, mediante un porcentaje válido del (71.3\%).

Esto concuerda bastante con el uso que los estudiantes de la UNED dicen hacer sobre la preparación de sus asignaturas, ya que un porcentaje elevado de ellos (62.8\%), afirman aplicarlas en la preparación de las mismas, lo cuál nos indica la necesidad de ir paulatinamente aproximando la docencia a distancia a este tipo de comunicación tecnológica donde su potencial de diálogo la hacen una opción adecuada en la estructura de la Universidad a Distancia como es la UNED. 


\section{CONCLUSIONES}

En el presente estudio se ha pretendido ofrecer una visión global de lo que es este tipo de enseñanza considerando, tanto algunos aspectos generales de la educación a distancia como otros más específicos de suma relevancia en el desarrollo de los procesos educativos, como son las Tecnologías de la Información y la Comunicación (TIC).

Se ha destacado el papel relevante que juega la UNED en el desarrollo y triunfo de la metodología de enseñanza a distancia, siendo una de las universidades a distancia de carácter unimodal pionera en la Unión Europea que ha sabido adaptarse a los avances tecnológicos, consiguiendo sobrevivir en periodos de crisis y de enormes dificultades, como las actuales, ofreciendo a la sociedad, tanto española como extranjera (sobre todo iberoamericana), los medios suficientes para la enseñanza y el aprendizaje.

En el modelo de enseñanza propuesto por la UNED, la tutoría, o clase tutorial, se establece como el enlace natural y efectivo de la calidad de su educación universitaria a distancia entre el profesor de la sede central y los estudiantes a través de la figura del profesor tutor del centro asociado. La tutoría, las unidades didácticas, la guía didáctica y de carrera y los medios audiovisuales, conforman el modelo de la enseñanza de la UNED, uno de los más completos de enseñanza universitaria a distancia en el mundo.

Por su ineludible relación íntima con la tutoría, la figura del centro asociado a la UNED cobra una importancia capital ya que posibilita, junto con la sede central, el que esta universidad imparta satisfactoriamente la enseñanza a distancia a sus cerca de doscientos cincuenta mil estudiantes.

Con la integración de la enseñanza de las TIC, se intentan determinar, a través de la participación de los estudiantes, las necesidades más demandadas en los centros asociados a la UNED y en la propia UNED (sede central).

\section{NOTAS}

1. Según García Aretio, (1986), el 20 de marzo de 1828 aparece un anuncio en la Gaceta de Boston ofreciendo material de enseñanza y tutorías por correspondencia.

2. Indicó la necesidad de "aprovechar la experiencia colectiva y la utilización compartida de recursos escasos en el campo de las nuevas tecnologías de la información, (NTI), aplicadas a la educación”. (Primer Congreso celebrado en París en 1989). Posteriormente en su segundo Congreso Internacional (celebrado en Moscú 
en julio de 1996), EDUCACIÓN E INFORMÁTICA, se analizaron las experiencias internacionales sobre la utilización de las NTI.

3. Para Van Dusen, (1997) el Campus Virtual, metáfora del enclave de enseñanza, aprendizaje e investigación creado por la convergencia de las TIC, es un complemento del curso presencial, sin ser un curso online.

4. Se entiende por "Red", según la Real Academia de la Lengua Española (2001), "el conjunto de ordenadores o de equipos informáticos conectados entre sí que pueden intercambiar información".

5. WebCT (Web Course Tools, o Herramientas para Cursos Web). Originalmente desarrollado en la Universidad de Columbia Británica, en Canadá por un miembro de la Escuela de Ingeniería en Informática, Murray Goldberg.

6. Productos desarrollados bajo la licencia GPL (General Public License) que tienen además la ventaja de ser libres, gratuitos y abiertos.

7. AVIP - Herramienta AudioVisual sobre tecnología IP, que permite desde cualquier punto con acceso a Internet, que los usuarios puedan acceder a los seminarios y tutorías tanto en directo como en diferido.

8. En el $\alpha$ de Cronbach la fiabilidad depende de la longitud de la prueba y de la covarianza entre sus ítems, lo que significa que el coeficiente mide la fiabilidad del test, o del instrumento, en función de dos.

\section{REFERENCIAS BIBLIOGRÁFICAS}

Arafeh, S. (2004). The implications of information and communications technologiesfor distance education: Final Report, SRI International \& American Institutes for Research. SRI Project Number P11913. [en línea] Disponible en: http:// www.sri.com/policy/csted/reports/ sandt/it/distance ED Lit review FINAL 6-9-04.pdf [consulta 2012, 12 de julio].

Area, M.; otros (2004). ¿Qué aporta INTERNET al cambio pedagógico en la educación superior? Universidad de La Laguna. En Pérez, R. (Coord.): Redes multimedia y diseños virtuales. Actas del III Congreso Internacional de Comunicación, Tecnología y Educación. Universidad de Oviedo. Septiembre, (128-135). [en línea] Disponible en: http://webpages.ull.es/users/manarea/Documentos/ documento7.htm [consulta 2012, 11 de mayo]
Bates, W. (2001). Como gestionar el cambio tecnológico. Estrategias para los responsables de centros universitarios. Barcelona: Gedisa.

Caballero Carrillo, R. (1997). La educación a distancia: una tecnología para la eficiencia de la educación. La Habana.

Cabero, J. (2001). Tecnología educativa. Diseño y utilización de medios en la enseñanza. Barcelona: Paidós.

Carmolinga, M.; Mendoza, N. E.; Maldonado, G. (2008). Principales tecnologías utilizadas en procesos de enseñanzaaprendizaje en educación superior. $R e$ vista de la Universidad Cristóbal Colón, 17-18 [en línea] Disponible en: http:// www.eumed.net/rev/rucc/17-18/mec. thm [consulta 2012, 11 de mayo].

Castell, M. (2001). La era de la información. Vol. I. La sociedad red. Madrid: Alianza Editorial. 
Comisión Europea (1998). First monitoring report on universal service in telecommunications in the Europea Union. Comunicación de la Unión Europea al Parlamento, al Consejo y al Comité de las Regiones COM (98) 182 Final.

Contreras Lara, M. E. (2004). Las instituciones de educación superior ante las nuevas tecnologías de información y comunicación. III Simposio Virtual de Computación en la Educación. [en línea]. Disponible en: htpp//www.informaticaeducativa.comvirtual2004/ponencias/formación/ContrerasLaraVega Ma.rtf [consulta 2012, 11 de mayo].

Cronbach, L. J. (1951). Coefficient alpha and the internal structure of test. Psychometrica, 16 (2), (297-334).

Domínguez Figaredo, D. (2009). La organización RED en educación a distancia. RIED. Revista Iberoamericana de educación a distancia, 12 (1), (15-36).

Fainholc, B. (2005). El uso inteligente de las TIC'S para una práctica educativa socioeducativa de calidad. Revista Latinoamericana de tecnología educativa, 4 (2) 53-63. [en línea] htpp://www.unex.es/ didactica/RELATEC/sumario 4 2:htm. [consulta 2012, 11 de mayo]

Fernández Aedo, R.; Felipe Panadeiro, A. (2009). Influencias de las tecnologías de la información y las comunicaciones en la universalización de la enseñanza. RIED. Revista Iberoamericana de Educación a Distancia, 12 (1), (63-75).

García Aretio, L. (1986). El origen de las Universidades a Distancia. Madrid: UNED.

García Aretio, L. (2007). La Universidad Nacional de Educación a Distancia (UNED) de España. RIED- Revista Iberoamericana de Educación a Distancia, 9, (1751).

García Aretio, L. (Coord.); Ruíz Corbella, M.; Domínguez Figaredo, D. (2007). De la educación a distancia a la educación virtual. Barcelona: Ariel.
García Llamas, J. L.; Pérez Juste, R.; Río Sadornil, D. (2003). Problemas y diseños de investigación resueltos. Madrid: Dykinson.

George, D.; Mallery, P. (1995). SPPS/PC + Step by Step: A Simple Guide and Referente. Wadsworth Publisinq Company.

George, D.; Mallery, P. (2006). SPPS for Windows Step by Step: A Simple Guide and Reference 13.0 Update. (6 $6^{\text {th }}$ ed.). United States: Pearson.

Grady, K. E.; Wallston, B. S. (1988). Research in health care setting. Newbury Park; Sage.

Martín, Q.; Cabero, M.; De Paz, Y. (2008). Tratamiento estadístico de datos con SPSS. Universidad de Salamanca. Ed. Thomson.

Marín Ibañez, R. (1995). El sistema multimedia de la enseñanza a distancia. Madrid: UNED.

Medina, A.; Sevillano, M. (1996). Nuevas tecnologías en la educación a distancia. En Tejedor; Valcárcel (Eds.). Perspectivas de las nuevas tecnologías en educación, (153-173). Madrid: Ed. Nárcea.

Moguel, S.; Alonzo, D. (2009). Dimensiones del aprendizaje y el uso de las TIC'S. El caso de la Universidad Autónoma de Campeche (México). RIED-Revista Iberoamericana de Educación a Distancia, 12 (1), (195-211).

Nunnaly, J. C. (1978). Psychometric theory. New Cork, USA: Mc Graw-Hill.

Ortíz, J. R. (1998). La educación a distancia en el umbral del nuevo paradigma telemático. Comunicación en el IV Congreso $R A B I E$, Brasilia. [en línea]. Disponible en: http://Ism.dei.uc.pt/ribie/docfiles/ txt200342415634191. pdf. [consulta 2012, 12 de julio].

Perez Juste, R.; Martinez Aragón, L. (1997). Evaluación de centros y calidad educativa. Bogotá, Colombia: Editorial Cincel.

Sevillano García, Ma ${ }^{\mathrm{a}}$. L. (1998). Nuevas tecnologías, medios de comunicación y 
educación. Formación inicial y permanente del profesorado. Madrid: CCS.

Sheskin, D. J. (2004). Handbook of parametric and nonparametric satistical procederes. Washigton D.D.: Chapaman\&Hall.

Van Dusen, G. C. (1997). The Virtual Campus. A: ASHE-ERIC Higher Education Report, 25 (5). Washington. D.F.: The George Washington University.
Williams, D. (2007). Keeping learning on track. In: FK Lester Jr. (Ed.). Second handbook of mathematics teaching and learning (1051-1098). Greenwich, CT: Information Age

Williams, V. (2000). Investigando Juntos. Siglo Cero, 31 (5), (5-9).

\section{PERFIL ACADÉMICO Y PROFESIONAL DE LOS AUTORES}

Emilia Carmena Yáñez. Licenciada en Ciencias Matemáticas por la Universidad Complutense de Madrid en las especialidades de Estadística e Investigación Operativa. Diplomada de la Escuela Estadística de la Universidad Complutense de Madrid en la especialidad de Estadística. Profesora agregada de Formación Profesional. Tutora del Centro Asociado de Madrid. Actualmente Profesora Titular de Escuela Universitaria e investigadora de la Universidad Nacional de Educación a Distancia, UNED.

E-mail: ecarmena@ccia.uned.es

Javier Navarro Fernández. Licenciado en Ciencias Matemáticas por la Universidad Complutense de Madrid en las especialidades de Estadística e Investigación Operativa. Diplomado de la Escuela Estadística de la Universidad Complutense de Madrid en la especialidad de Estadística. Profesor agregado de Instituto de Bachillerato. Tutor-Coordinador del Centro Asociado de Madrid. Doctor en Educación. Actualmente Profesor Titular de Universidad e Investigador de la Universidad Nacional de Educación a Distancia, UNED.

E-mail: jnavarro@ccia.uned.es

\section{DIRECCIÓN DE LOS AUTORES}

Facultad de Ciencias, UNED

Departamento de Estadística, Investigación Operativa y Cálculo Numérico, Senda del Rey 9, Madrid (28009), ESPAÑA. 
Fechas de recepción del artículo: 09/09/12

Fecha de aceptación del artículo: 22/01/13

\section{Como citar este artículo:}

Carmena Yáñez, E.; Navarro Fernández, J. (2012). Evolución del Uso de Tecnologías en la Enseñanza Universitaria a Distancia de la UNED. RIED. Revista Iberoamericana de Educación a Distancia, volumen 16, $\mathrm{n}^{0}$ 2, pp. 73-106. 